\title{
Iterative Dual Downlink Beamforming for Cognitive Radio Networks
}

\author{
Marius Pesavento Dana Ciochina Alex B. Gershman \\ Communication Systems Group, Technische Universität Darmstadt, Darmstadt, Germany
}

\begin{abstract}
We address the problem of multi-user downlink beamforming and power allocation in a cognitive radio (CR) secondary network (SN) with constraints on the total interference in the primary network (PN). We derive the Lagrange dual of the problem and show that both problems are equivalent. Two algorithms are proposed to solve the problem. The first is based on convex optimization and the second algorithm exploits the uplink-downlink duality that is enforced by the introduction of appropriate slack variables in the constrained optimization problem. This leads to a simple iterative technique that enjoys easy implementation and low computational costs. Simulation results illustrate that the proposed iterative technique converges to the global optimum in all cases.
\end{abstract}

\section{INTRODUCTION}

Cognitive radio (CR) has been identified as a promising path towards efficient usage of scarce radio resources. Secondary CR networks (CRNs) opportunistically access the licensed spectrum of the primary network ensuring that the latter is not adversely affected in its operation. In the underlay CRN, the secondary network obtains access to the licensed spectrum provided that the interference level caused to the primary network remains below some predefined interference temperature [1]. In the overlay systems context, PN and SN are allowed to cooperate and the interference level may be negotiated. A level-dependent licence fee applies to the SN in the latter case. In either CRN, an efficient control of the interference level caused by the SN using power allocation and beamforming techniques is of critical importance.

Optimum downlink power control and beamforming for multi-user cellular networks has recently received much attention [2]-[4]. In [5], the problem of minimizing the total transmit power subject to the quality-of-service (QoS) constraints on the primary users (PUs) is addressed and two equivalent solutions are provided that attain the optimal solution. The first algorithm is based on convex optimization, whereas the second one uses a simple iterative procedure. In the underlay CRN context, additional constraints on the total interference on the PUs need to be taken into account in the downlink beamforming problem. In [6] the secondary broadcast downlink beamforming problem has been addressed and various optimal formulations of the problem were investigated. Secondary multi-user downlink beamforming has been considered in [7], where supplementary inequality and robustness constraints have been incorporated into the generic convex optimizationbased approach of [5]. However, the latter approach is based on semi-definite relaxation (SDR). A substantial drawback of this method is that it requires to use interior-point algorithms for solving the resulting semi-definite programming problem. This generally increases the computational and implementational complexity when running the algorithm on real hardware or DSP architectures.

In this paper, we consider the problem of optimum power allocation and downlink beamforming in a cellular SN, where the secondary base station (BS) is required to meet the interference constraints imposed by the PN. This situation is, for example, encountered in femto- and picocells, where the home BS reuses the radio resources of the surrounding macro network, and the interference created by the $\mathrm{SN}$ to the macrocell needs to be controlled [8]. We show that for the downlink beamforming problem with interference constraints i) strong Lagrange duality holds and ii) a dual virtual uplink problem can be derived, that yields identical beamformers as for the original problem. Based on these results, two algorithms are proposed. The first algorithm is based on convex optimization and exploits the strong Lagrange duality of the problem considered. The second algorithm uses the uplinkdownlink duality to obtain the solution iteratively. The latter algorithm does not require any use of convex optimization software and enjoys simple implementation. Simulation results validate that our iterative algorithm converges to the optimal solution after a moderate number of iterations.

\section{Problem Formulation}

Let us consider a downlink CRN that involves $L$ PUs and $K$ secondary users (SUs). The SN BS contains $N_{t}$ transmit antennas and the PU and SU receivers are assumed to be single-antenna devices. Without any loss of generality, we assume that the users are indexed so that the users $1, \ldots, K$ are SUs, and the users $K+1, \ldots, K+L$ are PUs.

The signal transmitted at the SN BS is given by

$$
\boldsymbol{x}(n)=\boldsymbol{U} \operatorname{diag}\left\{\sqrt{p}_{1}, \ldots, \sqrt{p}_{K}\right\} \boldsymbol{s}(n)
$$

where $s(n) \triangleq\left[s_{1}(n), \ldots, s_{K}(n)\right]^{T}, s_{k}(n)$ denotes the symbol that is transmitted to the $k$ th SU at time $n, p_{k}$ denotes the transmitted power allocated to the $k$ th $\mathrm{SU}, \boldsymbol{U} \triangleq\left[\boldsymbol{u}_{1}, \ldots, \boldsymbol{u}_{K}\right]$ is the beamforming matrix, $\boldsymbol{u}_{k}$ denotes the normalized beamforming vector of the $k$ th $\mathrm{SU}, \operatorname{diag}\{\cdot\}$ denotes a diagonal matrix, and $(\cdot)^{T}$ stands for the transpose. Throughout the paper, we assume that the unit-norm constraint $\left\|\boldsymbol{u}_{k}\right\|=1$ holds for all the vectors $\boldsymbol{u}_{1}, \ldots, \boldsymbol{u}_{K}$, where $\|\cdot\|$ is the vector 2 -norm. The signal received at the $k$ th SU can be written as

$$
y_{k}(n)=\boldsymbol{h}_{k}^{T} \boldsymbol{x}(n)+z_{k}(n)
$$

where $z_{k}(n)$ is the additive white Gaussian sensor noise of variance $\sigma_{k}^{2}$, and $\boldsymbol{h}_{k} \triangleq\left[h_{k, 1}, \ldots, h_{k, N_{t}}\right]^{T}$ is the downlink channel 
vector of the $k$ th SU $(k=1, \ldots, K)$. Here, $h_{k, m}$ is the complex channel coefficient between the $m$ th transmit antenna of the SN $\mathrm{BS}$ and the $k$ th SU receiver. The interference signal received at the $l$ th PU can be written as

$$
y_{K+l}(n)=\boldsymbol{h}_{K+l}^{T} \boldsymbol{x}(n)
$$

where $\boldsymbol{h}_{K+l} \triangleq\left[h_{K+l, 1}, \ldots, h_{K+l, N_{t}}\right]^{T}$ is the downlink channel vector of the $l$ th PU $(l=1, \ldots, L)$, and $h_{K+l, m}$ is the complex channel coefficient between the $m$ th transmit antenna of the SN BS and the $l$ th PU receiver.

In the traditional beamforming setup, the objective of designing the beamforming matrix $\boldsymbol{U}$ and the downlink powers $p_{1}, \ldots, p_{K}$ is to minimize the total transmitted power $\sum_{k=1}^{K} p_{k}$ subject to some minimum QoS requirements for each user. This problem can be expressed as

$$
\begin{array}{ll} 
& \min _{\boldsymbol{U}, p_{1}, \ldots, p_{K}} \sum_{k=1}^{K} p_{k} \\
\text { s.t. } & \operatorname{SINR}_{k}^{\mathrm{DL}} \triangleq \frac{p_{k} \boldsymbol{u}_{k}^{H} \boldsymbol{R}_{k} \boldsymbol{u}_{k}}{\sum_{\substack{i \neq k \\
i=1}}^{K} p_{i} \boldsymbol{u}_{i}^{H} \boldsymbol{R}_{k} \boldsymbol{u}_{i}+\sigma_{k}^{2}} \geq \gamma_{k} ; k=1, \ldots, K \\
& p_{k} \geq 0 ;\left\|\boldsymbol{u}_{k}\right\|=1
\end{array}
$$

where the superscript "DL" denotes "downlink", $\gamma_{k}$ denotes the signal-to-interference-plus-noise-ratio (SINR) target and $\boldsymbol{R}_{k} \triangleq$ $\mathrm{E}\left\{\boldsymbol{h}_{k} \boldsymbol{h}_{k}^{H}\right\}$ is the downlink channel covariance matrix of the $k$ th user. In the CR framework, the total interference caused by the SN BS at the individual PUs needs to be controlled. In this case, we modify problem (4) by adding the following additional inequality constraints

$$
\mathrm{I}_{l}^{\mathrm{DL}} \triangleq \sum_{k=1}^{K} p_{k} \boldsymbol{u}_{k}^{H} \boldsymbol{R}_{K+l} \boldsymbol{u}_{k} \leq \frac{1}{\gamma_{K+l}} ; l=1, \ldots, L
$$

where $\boldsymbol{R}_{K+l} \triangleq \mathrm{E}\left\{\boldsymbol{h}_{K+l} \boldsymbol{h}_{K+l}^{H}\right\}$ is the downlink channel covariance matrix for the $l$ th $\mathrm{PU}$ and $1 / \gamma_{K+l}$ is the threshold for the interference level $\mathrm{I}_{l}^{\mathrm{DL}}$ of this user.

The duality results derived in this paper are based on the relative SINR balancing property, which is established when all inequality constraints in (4) and (5) are active, i.e., when strict equalities hold at the optimum. This is, for example, the case in the conventional downlink beamforming without PU interference constraints [4]-[5]. To see that this property holds, assume without loss of generality that at its optimum the relative SINR of the $k$ th $\mathrm{SU}, \mathrm{SINR}_{k}^{\mathrm{DL}} / \gamma_{k}$, is strictly larger than one. It is then always possible to reduce the relative SINR of this SU by scaling down the power allocated to it, and, therefore, to further reduce the objective function. At the same time, the relative SINR of the remaining SUs is increased so that none of the inequality constraints is violated. All these facts contradict to the optimality assumption. Hence, at the optimum point all SU constraints are satisfied with equality.

However, in the presence of the PUs the balancing property does not necessarily hold for the inequality constraints in (5), which may thus be inactive at the optimum. In this paper, we propose to incorporate new slack variables $p_{K+1}, \ldots, p_{K+L}$ in the underlying problem, so that the PU interference constraints in (5) transform to expressions similar to the active SU interference constraints in (4). Towards this aim, let us first invert the constraints in (5) and rewrite the optimization problem (4)-(5) as

$$
\begin{array}{ll} 
& \min _{\boldsymbol{U}, p_{1}, \ldots, p_{K}} \sum_{k=1}^{K} p_{k} \\
\text { s.t. } & \operatorname{SINR}_{k}^{\mathrm{DL}} \triangleq \frac{p_{k} \boldsymbol{u}_{k}^{H} \boldsymbol{R}_{k} \boldsymbol{u}_{k}}{\sum_{\substack{i \neq k \\
i=1}}^{K} p_{i} \boldsymbol{u}_{i}^{H} \boldsymbol{R}_{k} \boldsymbol{u}_{i}+\sigma_{k}^{2}} \geq \gamma_{k} ; k=1, \ldots, K \\
& \frac{1}{\mathrm{I}_{l}^{\mathrm{DL}}}=\frac{1}{\sum_{k=1}^{K} p_{k} \boldsymbol{u}_{k}^{H} \boldsymbol{R}_{K+l} \boldsymbol{u}_{k}} \geq \gamma_{K+l} ; l=1, \ldots, L \\
& p_{k} \geq 0 ;\left\|\boldsymbol{u}_{k}\right\|=1 .
\end{array}
$$

Next, let us introduce the slack variables $p_{K+l} \leq 1$ for $l=$ $1, \ldots, L$, that can be interpreted as the virtual downlink powers of the PUs. Replacing the numerator of the $l$ th constraint in (6) by the corresponding virtual downlink power $p_{K+l}$, we obtain the following optimization problem:

$$
\begin{array}{ll} 
& \min _{\boldsymbol{U}, p_{1} \ldots, p_{K+L}} \sum_{k=1}^{K} p_{k} \\
\text { s.t. } & \mathrm{SINR}_{k}^{\mathrm{DL}} \triangleq \frac{p_{k} \boldsymbol{u}_{k}^{H} \boldsymbol{R}_{k} \boldsymbol{u}_{k}}{\sum_{\substack{i \neq k \\
i=1}}^{K} p_{i} \boldsymbol{u}_{i}^{H} \boldsymbol{R}_{k} \boldsymbol{u}_{i}+\sigma_{k}^{2}} \geq \gamma_{k} ; k=1, \ldots, K \\
& \mathrm{SINR}_{K+l}^{\mathrm{VDL}} \triangleq \frac{p_{K+l}}{\sum_{k=1}^{K} p_{k} \boldsymbol{u}_{k}^{H} \boldsymbol{R}_{K+l} \boldsymbol{u}_{k}} \geq \gamma_{K+l} ; l=1, \ldots, L \\
& p_{k} \geq 0 ; \quad p_{K+l} \geq 0 ; p_{K+l} \leq 1 ;\left\|\boldsymbol{u}_{i}\right\|=1 .
\end{array}
$$

Here, $\operatorname{SINR}_{K+l}^{\mathrm{VDL}}$ denotes the virtual downlink SINR of the $l$ th PU. It is easy to see that problems (6) and (7) yield the same solution. With the introduction of the virtual PU downlink powers in (7), the PU constraints are transformed to a structure similar to the SU QoS constraints in (4). Recall that for latter constraints strict equality holds at the optimal solution. Following the similar argument, it can be shown that at the optimum of (7) the PU constraints are active and, therefore, can be replaced by equalities.

\section{UPLINK-DOWNLINK DUALITY}

In this section, we obtain an equivalent virtual uplink (VUL) problem that can be viewed as the dual of the cognitive downlink problem in (6). This problem is given by

$$
\begin{array}{ll} 
& \min _{\boldsymbol{U}, q_{1}, \ldots, q_{K+L}} \sum_{k=1}^{K} \gamma_{k} \sigma_{k}^{2} q_{k}-\sum_{l=1}^{L} p_{K+l} q_{K+l} \\
\text { s.t. } & \operatorname{SINR}_{k}^{\mathrm{VUL}}=\frac{q_{k} \boldsymbol{u}_{k}^{H} \boldsymbol{R}_{k} \boldsymbol{u}_{k}}{\boldsymbol{u}_{k}^{H}\left(\sum_{\substack{i=1 \\
i \neq k}}^{K+L} q_{i} \gamma_{i} \boldsymbol{R}_{i}+\boldsymbol{I}\right) \boldsymbol{u}_{k}} \geq 1 ; k=1, \ldots, K \\
& q_{k} \geq 0 ; q_{K+l} \geq 0 ; p_{K+l} \leq 1 ;\left\|\boldsymbol{u}_{k}\right\|=1 ; l=1, \ldots, L
\end{array}
$$

where $q_{1}, \ldots, q_{K}$ and $q_{K+1}, \ldots, q_{K+L}$ are the virtual uplink powers corresponding to the SUs and PUs, respectively.

The following lemma relates the virtual uplink problem to the original CR downlink problem.

Lemma 1: The downlink problems (6) and (7) and the dual virtual uplink problem (8) are equivalent in the sense that all 
of them have the same solution for the beamforming weight vectors $\boldsymbol{u}_{1}^{\star}, \ldots, \boldsymbol{u}_{K}^{\star}$, and attain the same objective function value at their optima.

Proof of Lemma 1: Define $\boldsymbol{p}_{1} \triangleq\left[p_{1}, \ldots, p_{K}\right]^{T}$ and $\boldsymbol{p}_{2} \triangleq$ $\left[p_{K+1}, \ldots, p_{K+L}\right]^{T}$ containing the downlink powers and the virtual downlink powers of the $K$ SUs and the $L$ PUs, respectively. Then, we can equivalently write the optimization problem (7) as

$$
\begin{array}{ll} 
& \min _{\boldsymbol{U}, \boldsymbol{p}_{1}, \boldsymbol{p}_{2}} \mathbf{1}_{K \times 1}^{T} \boldsymbol{p}_{1} \\
\text { s.t. } & \left(\boldsymbol{D}-\boldsymbol{G}_{1}\right) \boldsymbol{p}_{1}=\boldsymbol{\eta}_{1} ; \boldsymbol{p}_{1} \geq \mathbf{0}_{K \times 1} \\
& \boldsymbol{G}_{2} \boldsymbol{p}_{1}=\boldsymbol{p}_{2} ; \boldsymbol{p}_{2} \leq \mathbf{1}_{L \times 1} ;\left\|\boldsymbol{u}_{k}\right\|=1 ; k=1, \ldots, K
\end{array}
$$

where $\mathbf{1}_{K \times 1}$ is the $K \times 1$ vector containing ones in all its entries and

$$
\begin{aligned}
& {[\boldsymbol{D}]_{k, k} \triangleq \boldsymbol{u}_{k}^{H} \boldsymbol{R}_{k} \boldsymbol{u}_{k} ;\left[\boldsymbol{G}_{2}\right]_{l, j} \triangleq \gamma_{l} \boldsymbol{u}_{j}^{H} \boldsymbol{R}_{K+l} \boldsymbol{u}_{j}} \\
& {\left[\boldsymbol{G}_{1}\right]_{i, j} \triangleq \begin{cases}0, & i=j ; \\
\gamma_{i} \boldsymbol{u}_{j}^{H} \boldsymbol{R}_{i} \boldsymbol{u}_{j}, & i \neq j\end{cases} } \\
& \boldsymbol{\eta}_{1} \triangleq\left[\gamma_{1} \sigma_{1}^{2}, \ldots, \gamma_{K} \sigma_{K}^{2}\right]^{T}
\end{aligned}
$$

for all $l=1, \ldots, L$ and $k, i, j=1, \ldots, K$.

Let $\lambda_{\max }\{\cdot\}$ denote the maximum eigenvalue of a matrix. It can be shown that for $\lambda_{\max }\left\{\boldsymbol{D}^{-1} \boldsymbol{G}_{1}\right\}<1$ the matrix $\left(\boldsymbol{D}-\boldsymbol{G}_{1}\right)$ is invertible and that all entries of its inverse are nonnegative [5]. In this case, the vectors $\boldsymbol{p}_{1}=\left(\boldsymbol{D}-\boldsymbol{G}_{1}\right)^{-1} \boldsymbol{\eta}_{1}$ and $\boldsymbol{p}_{2}=$ $\boldsymbol{G}_{2} \boldsymbol{p}_{1}$ also contain only nonnegative elements and the power constraint $\boldsymbol{p}_{1} \geq \mathbf{0}_{K \times 1}$ in (9) can be replaced by a constraint on the maximum eigenvalue of the matrix $\boldsymbol{D}^{-1} \boldsymbol{G}_{1}$ [5]. The problem (9) then reads as

$$
\begin{array}{ll} 
& \min _{\boldsymbol{U}, \boldsymbol{p}_{1}, \boldsymbol{p}_{2}} \mathbf{1}_{K \times 1}^{T} \boldsymbol{p}_{1} \\
\text { s.t. } & \left(\boldsymbol{D}-\boldsymbol{G}_{1}\right) \boldsymbol{p}_{1}=\boldsymbol{\eta}_{1} ; \boldsymbol{G}_{2} \boldsymbol{p}_{1}=\boldsymbol{p}_{2} \\
& \lambda_{\max }\left\{\boldsymbol{D}^{-1} \boldsymbol{G}_{1}\right\}<1 ; \boldsymbol{p}_{2} \leq \mathbf{1}_{L \times 1} ;\left\|\boldsymbol{u}_{k}\right\|=1 ; k=1, \ldots, K .
\end{array}
$$

We further define the vectors

$$
\boldsymbol{q}_{1} \triangleq\left[q_{1}, \ldots, q_{K}\right]^{T}=\left(\boldsymbol{D}-\boldsymbol{G}_{1}\right)^{-T}\left(\mathbf{1}+\boldsymbol{G}_{2}^{T} \boldsymbol{q}_{2}\right)
$$

and $\boldsymbol{q}_{2} \triangleq\left[q_{K+1}, \ldots, q_{K+L}\right]^{T}$ and expand the cost function (13) with the term $\boldsymbol{p}_{2}^{T} \boldsymbol{q}_{2}=\boldsymbol{\eta}_{1}^{T}\left(\boldsymbol{D}-\boldsymbol{G}_{1}\right)^{-T} \boldsymbol{G}_{2}^{T} \boldsymbol{q}_{2}$, which is obtained directly from the constraints in (13). The problem (13) is then equivalent to

$$
\begin{array}{ll} 
& \min _{\boldsymbol{U}, \boldsymbol{p}_{1}, \boldsymbol{p}_{2}} \boldsymbol{p}_{1}^{T} \mathbf{1}_{K \times 1}+\boldsymbol{\eta}_{1}^{T}\left(\boldsymbol{D}-\boldsymbol{G}_{1}\right)^{-T} \boldsymbol{G}_{2}^{T} \boldsymbol{q}_{2}-\boldsymbol{p}_{2}^{T} \boldsymbol{q}_{2} \\
\text { s.t. } & \left(\boldsymbol{D}-\boldsymbol{G}_{1}\right) \boldsymbol{p}_{1}=\boldsymbol{\eta}_{1} ; \boldsymbol{G}_{2} \boldsymbol{p}_{1}=\boldsymbol{p}_{2} \\
& \lambda_{\max }\left\{\boldsymbol{D}^{-1} \boldsymbol{G}_{1}\right\}<1 ;\left\|\boldsymbol{u}_{k}\right\|=1 ; \boldsymbol{p}_{2} \leq \mathbf{1}_{L \times 1} ; k=1, \ldots, K .
\end{array}
$$

Inserting $\boldsymbol{q}_{1}$ from (14) and $\boldsymbol{p}_{1}=\left(\boldsymbol{D}-\boldsymbol{G}_{1}\right)^{-1} \boldsymbol{\eta}_{1}$ in the objective function of (15), we obtain

$$
\begin{array}{ll} 
& \min _{\boldsymbol{U}, \boldsymbol{q}_{1}, \boldsymbol{q}_{2}} \boldsymbol{\eta}_{1}^{T} \boldsymbol{q}_{1}-\boldsymbol{p}_{2}^{T} \boldsymbol{q}_{2} \\
\text { s.t. } & \left(\boldsymbol{D}-\boldsymbol{G}_{1}^{T}\right) \boldsymbol{q}_{1}-\boldsymbol{G}_{2}^{T} \boldsymbol{q}_{2}=\mathbf{1} \\
& \lambda_{\max }\left\{\boldsymbol{D}^{-1} \boldsymbol{G}_{1}\right\}<1 ; \boldsymbol{p}_{2} \leq \mathbf{1}_{L \times 1} ;\left\|\boldsymbol{u}_{k}\right\|=1 ; k=1, \ldots, K
\end{array}
$$

where the first constraint in (16) follows directly from (14).

Note next that $\lambda_{\max }\left\{\boldsymbol{D}^{-1} \boldsymbol{G}_{1}\right\}=\lambda_{\max }\left\{\boldsymbol{D}^{-1} \boldsymbol{G}_{1}^{T}\right\}<1$ implies that the Neumann series $\left(\boldsymbol{I}-\boldsymbol{D}^{-1} \boldsymbol{G}_{1}^{T}\right)^{-1}=\sum_{m=0}^{\infty}\left(\boldsymbol{D}^{-1} \boldsymbol{G}_{1}^{T}\right)^{m}$ converges to a matrix with non-negative entries. If we choose $\boldsymbol{q}_{2} \geq \mathbf{0}_{L \times 1}$ and if the constraints in (16) are satisfied, it follows that $\boldsymbol{q}_{1} \geq \mathbf{0}_{K \times 1}$. This is due to the fact that in this case, all the vectors and matrices in (14), including the inverse $\left(\boldsymbol{D}-\boldsymbol{G}_{1}\right)^{-T}=\boldsymbol{D}^{-1}\left(\boldsymbol{I}-\boldsymbol{D}^{-1} \boldsymbol{G}_{1}^{T}\right)^{-T}$ have non-negative entries. The optimization problem (16) then becomes equivalent to

$$
\begin{array}{ll} 
& \min _{\boldsymbol{U}, \boldsymbol{q}_{1}, \boldsymbol{q}_{2}} \boldsymbol{\eta}^{T} \boldsymbol{q}_{1}-\boldsymbol{p}_{2}^{T} \boldsymbol{q}_{2} \\
\text { s.t. } & \left(\boldsymbol{D}-\boldsymbol{G}_{1}^{T}\right) \boldsymbol{q}_{1}-\boldsymbol{G}_{2}^{T} \boldsymbol{q}_{2}=\mathbf{1} ;\left\|\boldsymbol{u}_{k}\right\|=1 ; k=1, \ldots, K \\
& \boldsymbol{q}_{1} \geq \mathbf{0}_{K \times 1} ; \boldsymbol{q}_{2} \geq \mathbf{0}_{L \times 1} ; \boldsymbol{p}_{2} \leq \mathbf{1}_{L \times 1} .
\end{array}
$$

This problem defines the virtual uplink beamforming problem of (8) in the matrix notation.

\section{LAGRANGE DUALITY}

The Lagrange dual problem corresponding to the cognitive downlink problem (6) can be formulated as

$$
\begin{aligned}
& \max _{q_{1}, \ldots, q_{K+L}} \sum_{k=1}^{K} \gamma_{k} \sigma_{k}^{2} q_{k}-\sum_{l=1}^{L} q_{K+l} \\
& \text { s.t. } \quad \boldsymbol{M}_{k}=\boldsymbol{I}-q_{k} \boldsymbol{R}_{k}+\sum_{\substack{i \neq k \\
i=1}}^{K+L} \gamma_{i} q_{i} \boldsymbol{R}_{i} \geqslant 0 ; k=1, \ldots, K
\end{aligned}
$$

where $q_{1}, \ldots, q_{K+L} \geq 0$ denote the Lagrange multipliers [9].

Lemma 2: For the Lagrange dual problem (18), strong duality holds. That is, the duality gap between the cognitive downlink problem (6) and the Lagrange dual problem (18) is zero, and both optimization problems attain the same objective function value at their optima. Further, the optimum beamforming weight vectors $\boldsymbol{u}_{1}^{\star}, \ldots, \boldsymbol{u}_{K}^{\star}$ of (6) are given by the set of vectors in the nullspace of the corresponding semidefinite matrices $\boldsymbol{M}_{1}, \ldots, \boldsymbol{M}_{K}$ in (18) evaluated at the optimum Lagrange multipliers $q_{1}^{\star}, \ldots, q_{K+L}^{\star}$.

Proof of Lemma 2: Let $\boldsymbol{u}_{k}^{\star}, p_{m}^{\star}$ and $q_{m}^{\star}$ denote the optimum beamforming vector, downlink power, and uplink power, respectively, that solve the problem (7) and (8) for $k=1, \ldots, K$ and $m=1, \ldots, K+L$. Assume without loss of generality that at the optimum all the PU interference constraints in (5) are satisfied with strict inequality. Then there exist scalars $\alpha_{l}^{\star}>1$ for $l=1, \ldots, L$, such that

$$
\gamma_{K+l}^{\star}=\frac{1}{\sum_{k=1}^{K} p_{k}^{\star} \boldsymbol{u}_{k}^{\star H} \boldsymbol{R}_{K+l} \boldsymbol{u}_{k}^{\star}}=\alpha_{l}^{\star} \gamma_{K+l}>\gamma_{K+l} .
$$

Note that in this case the PU SINR target $\gamma_{l}$ in (6) can be replaced by the enhanced target $\alpha_{l}^{\star} \gamma_{l}$ without changing the optimal solution or violating any constraint of the problem (6). If we now consider the virtual uplink problem (8) and substitute $\gamma_{K+l}^{(\alpha)}=\alpha_{l}^{\star} \gamma_{K+l}, p_{K+l}^{(\alpha)}=\alpha_{l}^{\star} p_{K+l}, q_{K+l}^{(\alpha)}=q_{K+l} / \alpha_{l}^{\star}$, for $l=1, \ldots, L$, then it follows from the PU constraints in (7) and (19) that $p_{K+l}^{(\alpha)}=1$ at the optimum. Note that this also holds true if the interference constraint (5) of a particular PU (e.g., the $l$ th user) is satisfied with equality at the optimum. In this case, we define $\gamma_{K+l}^{(\alpha)}=\gamma_{K+l}$ and $\alpha_{K+l}^{\star}=1$, so that according to the PU constraints in (6) and (7) $p_{K+l}^{(\alpha)}=1$ and $q_{K+l}^{(\alpha)}=q_{K+l}$. 
If we insert these substitutions into (8) and set $\gamma_{k}=\gamma_{k}^{(\alpha)}$ and $q_{k}=q_{k}^{(\alpha)}$ for $k=1, \ldots, K$, then we have

$$
\begin{array}{ll} 
& \min _{\substack{(\alpha) \\
, q_{1}^{(\alpha)}, q_{K+L}^{(\alpha)}}} \sum_{k=1}^{K} \gamma_{k}^{(\alpha)} \sigma_{k}^{2} q_{k}^{(\alpha)}-\sum_{l=1}^{L} q_{K+l}^{(\alpha)} \\
\text { s.t. } & \frac{q_{k}^{(\alpha)} \boldsymbol{u}_{k}^{H} \boldsymbol{R}_{k} \boldsymbol{u}_{k}}{} \\
& \boldsymbol{u}_{k}^{H}\left(\sum_{\substack{i \neq k \\
i=1}}^{K+L} q_{i}^{(\alpha)} \gamma_{i}^{(\alpha)} \boldsymbol{R}_{i}+\boldsymbol{I}\right) \boldsymbol{u}_{k} \\
& q_{k}^{(\alpha)} \geq 0 ; q_{K+l}^{(\alpha)} \geq 0 ; p_{K+l}^{(\alpha)} \leq 1 ;\left\|\boldsymbol{u}_{k}\right\|=1 ; l=1, \ldots, K .
\end{array}
$$

Similarly, using the same substitutions as above in the Lagrange dual problem (18), we have

$$
\begin{array}{ll} 
& \max _{\substack{(\alpha) \\
q_{1}^{(\alpha)}, q_{K+L}^{(\alpha)}}} \sum_{k=1}^{K} \gamma_{k}^{(\alpha)} \sigma_{k}^{2} q_{k}^{(\alpha)}-\sum_{l=1}^{L} q_{K+l}^{(\alpha)} \\
\text { s.t. } & \boldsymbol{I}-q_{k}^{(\alpha)} \boldsymbol{R}_{k}+\sum_{\substack{i \neq k \\
i=1}}^{K+L} \gamma_{i}^{(\alpha)} q_{i}^{(\alpha)} \boldsymbol{R}_{i} \geqslant 0 ; k=1, \ldots, K \\
& q_{k}^{(\alpha)} \geq 0 ; q_{K+l}^{(\alpha)} \geq 0 ; l=1, \ldots, L .
\end{array}
$$

It can be shown [5], that the virtual uplink dual problem in (20), and the Lagrange dual problem (21) have identical solutions taken from the set

$$
\begin{aligned}
& \boldsymbol{M}_{k}^{(\alpha)}=\boldsymbol{I}-q_{k}^{(\alpha)} \boldsymbol{R}_{k}+\sum_{\substack{i \neq k \\
i=1}}^{K+L} \gamma_{i}^{(\alpha)} q_{i}^{(\alpha)} \boldsymbol{R}_{i} \geq 0 ; \operatorname{det}\left\{\boldsymbol{M}^{(\alpha)}\right\}=0 \\
& q_{k}^{(\alpha)} \geq 0 ; q_{K+l}^{(\alpha)} \geq 0 ; k=1, \ldots, K ; l=1, \ldots, L .
\end{aligned}
$$

Expression (22) defines a set of uplink powers $q_{1}^{(\alpha)}, \ldots, q_{K+L}^{(\alpha)}$ for which the matrices $\boldsymbol{M}_{k}^{(\alpha)}$ are non-negative definite and have at least one zero eigenvalue [5].

We note that both problems, the virtual uplink problem (20) and the Lagrange dual problem (21) have identical objective functions, with the difference that in the former problem the objective is minimized, while it is maximized in the latter problem. We conclude that both these problems have identical solutions $q_{1}^{\star}, \ldots, q_{K}^{\star}$ and attain the same objective function value at the optimum. If $\boldsymbol{u}_{1}^{\star}, \ldots, \boldsymbol{u}_{K}^{\star}$ denote the optimum beamforming weight vectors of the virtual uplink problem (20), then it is clear that these vectors lie in the nullspace of the semi-definite matrices $\boldsymbol{M}_{1}^{(\alpha)}, \ldots, \boldsymbol{M}_{K}^{(\alpha)}$ in (22) evaluated at $q_{1}^{\star}, \ldots, q_{K}^{\star}$. According to Lemma 1 , the primal problem (6) is equivalent to the virtual uplink problem (20). Thus, all three problems yield identical beamforming solutions $\boldsymbol{u}_{1}^{\star}, \ldots, \boldsymbol{u}_{K}^{\star}$ and strong Lagrange duality holds for problem (6). This further implies that the duality gap is zero.

It follows from the strong duality result of the Lemma 2, that the complementary slackness conditions $\left(\sum_{k=1}^{K} p_{k}^{\star} \boldsymbol{u}_{k}^{\star H} \boldsymbol{R}_{K+l} \boldsymbol{u}_{k}^{\star}-\gamma_{K+l}\right) q_{K+l}^{\star}=0$ of the Karush-KuhnTucker (KKT) conditions [9] for problem (6) hold at the optimum. With the constraints in (20), this means that $p_{K+l}^{\star}=1$ if the $l$ th PU constraint is active at the optimum, or the corresponding virtual uplink power $q_{K+l}=0$. The complementary slackness condition can thus be compactly written as $\left(p_{K+l}^{\star}-1\right) q_{K+l}^{\star}=0$.
It is worth noting that the same approach can be used to prove, from the uplink-downlink duality, that also the SDR approach of [7] yields optimal solutions in all cases. We stress that the latter fact was not proven in [7].

The Lagrange dual of any problem is always convex [9]. Lemma 2 states that the optimal beamformers for the cognitive downlink problem (6) can be obtained from the solution of the SDP problem in (18). Thus the first algorithm we propose consists of directly solving problem (18) using standard interior-point software [10]. The optimum Lagrange multipliers $q_{1}^{\star}, \ldots, q_{K+L}^{\star}$ and the optimum beamformers $\boldsymbol{u}_{1}^{\star}, \ldots, \boldsymbol{u}_{K}^{\star}$ are obtained from the vectors in the nullspace of the matrices on the left side of the semi-definiteness constraints in (18). The downlink powers are obtained, e.g., from the first constraints in (9), and equations (10)-(11). In this paper, however, we are interested in obtaining a computationally efficient algorithm that does not require interior-point software. Therefore, the SDP algorithm is used just as a performance benchmark for the iterative algorithm introduced in the next section.

\section{The iterative Algorithm}

Next, we develop a simple algorithm for solving the optimum downlink beamforming and power allocation problem (6) iteratively. This algorithm can be viewed as an nontrivial extension of the procedure in [5] for the case with PU interference constraints.

Initialization: Initialize $q_{m}(0)=1$, for $m=1, \ldots, K+L$.

Parameter update: For $t=1,2, \ldots$ until convergence, iterate the following steps:

1) Beamformer update: Find

$$
\mu_{k}(t+1)=\max _{\left\|\boldsymbol{u}_{k}\right\|=1} \frac{q_{k}(t) \boldsymbol{u}_{k}^{H} \boldsymbol{R}_{k} \boldsymbol{u}_{k}}{\boldsymbol{u}_{k}^{H}\left(\sum_{\substack{i \neq k \\ i=1}}^{K+L} q_{i}(t) \gamma_{i} \boldsymbol{R}_{i}+\boldsymbol{I}\right) \boldsymbol{u}_{k}}
$$

and the vector $\boldsymbol{u}_{k}$, for $k=1, \ldots, K$, from the solution of the corresponding generalized eigenproblem.

2) Virtual uplink power allocation update (SUs): Compute

$$
q_{k}(t+1)=q_{k}(t) / \mu_{k}(t+1)
$$

for $k=1, \ldots, K$.

3) Transformation to the downlink domain: For the beamforming vectors obtained in step 1 compute the equivalent virtual downlink powers $\boldsymbol{p}_{1}(t+1)=\left(\boldsymbol{D}-\boldsymbol{G}_{1}\right)^{-1} \boldsymbol{\eta}_{1}$ and $\boldsymbol{p}_{2}(t+1)=\boldsymbol{G}_{2} \boldsymbol{p}_{1}(t+1)$ according to the constraints in (9) with $\boldsymbol{D}, \boldsymbol{G}_{1}, \boldsymbol{G}_{2}$ and $\boldsymbol{\eta}_{1}$ defined in (10)-(12), respectively.

4) Virtual uplink power allocation update (PUs): Compute

$$
q_{K+l}(t+1)= \begin{cases}p_{K+l}(t+1) q_{K+l}(t), & \text { if } \min \left\{p_{l}, \ldots, p_{K}\right\} \geq 0 \\ q_{K+l}(t+1), & \text { otherwise. }\end{cases}
$$

Convergence check: The following conditions can be used for checking the convergence:

$$
\left|q_{k}(t)-q_{k}(t+1)\right| \leq \delta ; \quad p_{K+l}(t+1)-1 \leq \delta
$$

for $k \leq K+L$ and $l=1, \ldots, L$ and a small positive constant $\delta$. The optimum power allocations after convergence at iteration $T$ are given by $p_{1}(T), \ldots, p_{K}(T)$, and the corresponding beamforming vectors are obtained as $\boldsymbol{u}_{1}(T), \ldots, \boldsymbol{u}_{K}(T)$. 
This iterative algorithm has several interesting properties. First of all, the update procedure in step 2 stems from the observation that, according to the SU constraints in (8), the generalized eigenvalue $\mu_{k}(t)$ can be viewed as a measure of the instantaneous uplink SINR of the $k$ th $\mathrm{SU}, \mathrm{SINR}_{k}^{\mathrm{VUL}}$, that is achieved with a specific set of beamformers. The algorithm thus drives the SINR of the $k$ th SU towards the target $\mathrm{SINR}_{k}^{\mathrm{VUL}}=1$. Moreover, if at a specific iteration $p_{K+l}$ exceeds one (i.e., the solution is infeasible), then the virtual uplink power allocated to the $l$ th user is increased. This ensures that in the next iteration more emphasis is given to the interference suppression of the $l$ th PU in the virtual uplink beamformer design. The resulting reduction of the interference level, in turn, initiates some reduction of the slack variable $p_{K+l}$ to achieve the optimum balancing point. Conversely, if at some specific iteration $p_{K+l}<1$, then (25) reduces the value of $q_{K+l}$, and, thus less emphasis is given to the interference suppression of the $l$ th PU in the next iteration.

Another remarkable property of the uplink power updating procedures (24) and (25) is that the solution $q_{1}^{\star}, \ldots, q_{K+L}^{\star}$, $p_{1}^{\star}, \ldots, p_{K+L}^{\star}$ and $\boldsymbol{u}_{1}^{\star}, \ldots, \boldsymbol{u}_{K}^{\star}$ of the cognitive downlink beamforming problem (7) and the corresponding virtual uplink problem (8) are fixed points of the iteration in the sense that $q_{k}^{\star}=q_{k}^{\star} / \mu_{k}^{\star}$ and $q_{K+l}^{\star}=p_{K+l}^{\star} q_{K+l}^{\star}$ for $k=1, \ldots, K$ and $l=1, \ldots, L$. To see that this statement holds true, consider that at the optimum the relative SINR of the $k$ th user, given by $\mu_{k}$, is equal to one. Similarly, according to the complementary slackness condition derived in the previous section, at the optimum either $p_{K+l}^{\star}=1$ or $q_{K+l}^{\star}=0$ and thus $q_{K+l}^{\star}=p_{K+l}^{\star} q_{K+l}^{\star}$ is a fixed point of the iteration (25).

\section{Simulation Results}

In this section, we present simulation results illustrating the performance of the proposed iterative algorithm in comparison with the benchmark SDP technique (which, as has been proven above, is equivalent performance-wise to the SDR beamformer of [7]). The software package CVX has been used for solving the corresponding SDP problem. The case of $K=5$ SUs and $L=5$ PUs has been considered. The SN BS has been assumed to have $N_{t}=9$ antennas. Interference levels $1 / \gamma_{K+l}=-6 \mathrm{~dB}(l=1, \ldots, L)$ have been assumed for each PU. The QoS constraints $\gamma_{k}(k=1, \ldots, K)$ of the SUs were drawn from a uniform distribution in the interval $[0 \mathrm{~dB} ; 10 \mathrm{~dB}]$. Rayleigh fading channels and noise variances of $-6 \mathrm{~dB}$ were assumed. A total of $N=1000$ independent Monte-Carlo runs were performed, with independent channel realizations and different QoS targets drawn in every run. In Figure 1, the root mean-square estimation error (RMSE), $\sqrt{1 / N \sum_{n=1}^{N} \sum_{m=1}^{K+L}\left(p_{m}(T)-p_{m}^{\star}\right)^{2}}$, is displayed for for all channel realizations and for the best $95 \%$ and $98 \%$ of the channel realizations. Here, $p_{m}(T)$ is the downlink power obtained from our iterative algorithm after the $T$ th iteration, and $p_{m}^{\star}$ $(m=1, \ldots, K+L)$ are the optimal downlink power allocations obtained from our benchmark SDP algorithm. We observe that our iterative technique converges close to the optimal solution after a moderate number of iterations. According to Figure 1,

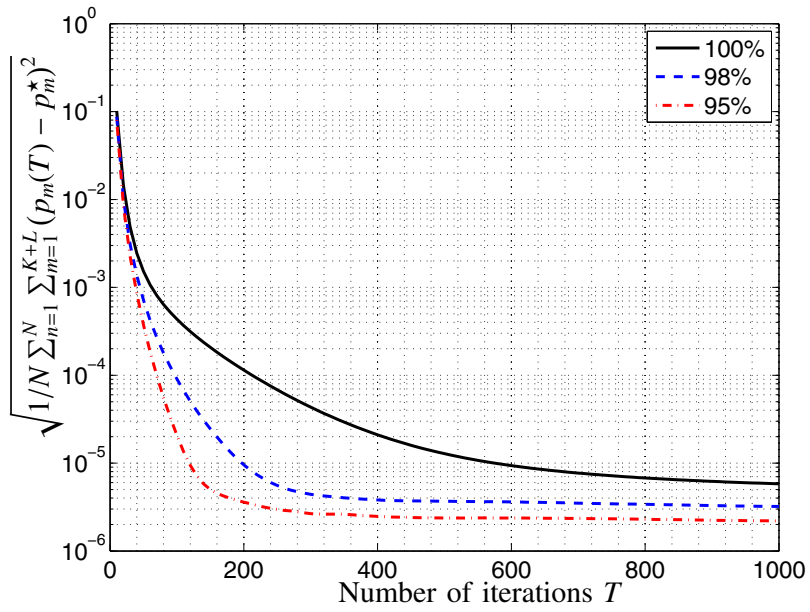

Fig. 1. RMSE of the downlink power versus number of iterations

if a few percents of outage is allowed, then the convergence speed can be substantially improved.

The complexity of the proposed iterative algorithm is $O\left\{K N_{t}^{2}\right\}$ per iteration. Note that the SDR algorithm in [7] has the complexity $O\left\{\left(K N_{t}\right)^{3}\right\}$ and requires at most $O\left\{\left(K N_{t}\right)^{0.5}\right\}$ iterations for convergence.

\section{AcKNOWLEDGEMENT}

This work has been supported in parts by the European Commission under FP7 Project CROWN, and the European Research Council (ERC) Advanced Investigator Grants Program under Grant 227477-ROSE.

\section{REFERENCES}

[1] S. Haykin, "Cognitive radio: Brain-empowered wireless communications," IEEE J. Sel. Areas Commun., vol. 23, no. 2, pp. 201-220, Feb. 2005

[2] F. Rashid-Farrokhi, L. Tassiulas, and K. J. R. Liu, "Joint optimal power control and beamforming in wireless networks using antenna arrays," IEEE Trans. Commun., vol. 46, no. 10, pp. 1312-1324, Oct. 1998.

[3] W. Yang and G. Xu, "Optimal downlink power assignment for smart antenna systems," in Proc. IEEE ICASSP, vol. 6, pp. 3337-3340, Seattle, WA, May 1998.

[4] M. Schubert and H. Boche, "Solution of the multiuser downlink beamforming problem with individual SINR constraints," IEEE Trans. Veh. Tech., vol. 53, no. 1, pp. 18-28, Jan. 2004.

[5] M. Bengtsson and B. Ottersten, "Optimal and suboptimal transmit beamforming," in Handbook of Antennas in Wireless Communications, L. Godara, Editor, Boca Raton, CRC, FL, Aug. 2001.

[6] K. T. Phan, S. A. Vorobyov, N. D. Sidiropoulos, and C. Tellambura, "Spectrum sharing in wireless networks via QoS-aware secondary multicast beamforming", IEEE Trans. on Signal Processing, vol. 57, no. 6, pp. 2323-2335, June 2009.

[7] K. Cumanan, R. Krishna, V. Sharma, and S. Lambotharan, "Robust interference control techniques for multiuser cognitive radios using worst-case performance optimization," Proc. of Asilomar Conf. on Sign., Syst., and Comp., Pacific Grove, USA, pp. 378-382, Nov. 2008.

[8] D. Calin, H. Claussen, and H. Uzunalioglu, "On femto deployment architectures and macrocell offloading benefits in joint macro-femto deployments," IEEE Commun. Magazine, vol. 48, no. 1, pp. 26-32, January 2010.

[9] S. Boyd and L. Vandenberghe, Convex Optimization, Cambridge University Press, 2004

[10] M. Grant and S. Boyd. CVX: Matlab software for disciplined convex programming. http://stanford.edu/ boyd/cvx, June 2009. 